\title{
AVALIAÇÄO DA CAPACIDADE FíSICA E DO ESTADO NUTRICIONAL EM CANDIDATOS AO TRANSPLANTE HEPÁTICO
}

\author{
Ana V. A. Leitão, Carmen L. N. Castro, tatianna M. Basile, thiago H. S. Souza, Valeria B. Braulio* \\ Trabalho realizado no Laboratório de Pesquisa em Capacidade Física, Função Muscular e Avaliação \\ Nutricional do Hospital Universitário Clementino Fraga Filho - Universidade Federal do Rio de Janeiro, RJ.
}

RESUMO - OBjETIVo. Avaliar o estado nutricional, os indicadores de capacidade física e suas relações com a função hepática, em pacientes candidatos ao transplante de fígado.

Métodos. Foram estudados 32 pacientes pré-transplante, classificados como Child-Pugh A (25\%), B (22\%) e C (53\%). A capacidade física foi avaliada pelos parâmetros: força muscular do quadríceps, dos flexores plantares e dorsais do pé; equilíbrio estático; coordenação; velocidade da marcha; teste de 6 minutos de caminhada e teste "timed get up and go"; índices de Barthel e de Karnofsky. $O$ estado nutricional foi avaliado pelo escore de Mendenhall e classificado segundo Blackburn.

Resultados. Foi detectado déficit de força de quadríceps ( $32 \%$ dos pacientes), do equilíbrio (34,8\%), da velocidade da marcha $(66,7 \%)$ e da coordenação (15,6\%). 0 teste de caminhada indicou baixo desempenho físico em $72,5 \%$ dos pacientes (moderado em
40,9\% e grave em $31,6 \%)$. 0 índice de Karnofsky revelou que 43,8\% dos pacientes necessitava assistência considerável. Foi identificada desnutrição em $62,5 \%$ dos pacientes (leve em $28,13 \%$; moderada e grave em 34,37\%). 0 teste do qui quadrado não mostrou associação entre a classificação de Child-Pugh e 0 estado nutricional, 0 teste de caminhada e 0 índice de Karnofsky. A associação entre 0 teste de caminhada e 0 índice de Karnofsky foi altamente significante $(\mathrm{p}<0,00 \mathrm{I})$.

Conclusão. Os resultados monstraram que a incapacidade física e a desnutrição foram altamente prevalentes nos pacientes estudados, independente do grau da disfunção hepática avaliada segundo a classificação tradicional de Child-Pugh.

UNITERMOS: Transplante hepático. Estado nutricional. Capacidade física. Classificação de Child-Pugh.

\section{INTRODUÇÃO}

Nos candidatos ao transplante de fígado, a doença hepática avançada é responsável por alterações metabólicas acompanhadas de desnutrição', perda da massa e função muscu$\operatorname{lar}^{2}$, com conseqüente deficiência motora global e inatividade física ${ }^{3,4}$, as quais interferem negativamente nas atividades da vida diária (AVD) e na qualidade de vida 4 .

O tempo de espera para o transplante favorece o aparecimento de condições mórbidas, decorrentes do agravamento da doença hepática, da desnutrição, do hipermetabolismo e da inatividade física, as quais irão afetar negativamente 0 prognóstico ${ }^{5}$. No entanto, poucos estudos têm investigado a

* Correspondência:

Serviço de Nutrologia - Hospital Universitário Clementino Fraga Filho $9^{\circ}$ andar, sala E- 14

Av Brigadeiro Trompovsky s/n, Cidade Universitária, Ilha do Fundão, CEP: 21941-590

Rio de Janeiro, RJ - Tel/Fax: 2562-2730 E-mail:bender@acd.ufri.br capacidade física e o grau de desempenho funcional na fase prévia ao transplante hepático ${ }^{3}$ ou na fase posterior à cirurgia ${ }^{4,6}$.

O objetivo do presente estudo foi avaliar 0 estado nutricional, os indicadores de função neuromotora, e as inter-relações entre estes parâmetros e o grau de comprometimento da função hepática, em pacientes candidatos ao transplante de fígado.

\section{Métodos}

Foram estudados 32 pacientes consecutivos (19 homens e 13 mulheres), com idade média de $47,8 \pm 15$ anos, inscritos no Programa de Transplante de Fígado do Hospital Universitário Clementino Fraga Filho da Universidade Federal do Rio de Janeiro. Os pacientes foram classificados quanto ao grau de comprometimento da função hepática, segundo a classificação de Child-Pugh ${ }^{7}$, cujos critérios estão descritos na Tabela I. Desse modo, $25 \%$ dos pacientes foram classificados como Child A, $22 \%$ como Child B e $53 \%$ como Child C. Em relação ao diagnóstico primário da doença, os pacientes apresentavam: hepatite crônica por vírus $C(n=17)$, hepatopatia colestática $(n=6)$, hepatite autoimune $(n=2)$, amiloidose $(n=2)$, hepatite por vírus $C(n=I)$, carcinoma hepatocelular $(n=I)$, cirrose alcoólica $(n=I)$, síndrome de Caroli $(n=I)$ e cirrose criptogênica $(n=I)$.

A avaliação funcional neuromotora foi realizada pelos seguintes testes: I) teste muscular manual $(\mathrm{TMM})^{8}$, para medir a força muscular do quadríceps; 2) teste de Perry', para avaliar a força dos músculos flexores plantares; 3 ) teste de caminhada nos calcanhares, para avaliar a força dos flexores dorsais do pél0 ;4) teste de equilíbrio estático em posição semi-tandem $^{\prime \prime}$; 5) teste de coordenação calcanharjoelho $\left.{ }^{11} ; 6\right)$ teste de velocidade da marcha ${ }^{12,13}$ $(\mathrm{m} / \mathrm{s})$, que é o tempo gasto em segundos para percorrer uma distância de 10 metros; 7 ) teste de tolerância ao exercício-teste de seis minutos de caminhada - de acordo com o protocolo de Guyatt ${ }^{14} ; 8$ ) teste "timed get up and go" ${ }^{\prime \prime}$, considerado normal o tempo inferior a 20 segundos $^{17}$. O teste de seis minutos de 


\begin{tabular}{lccc}
\hline \multicolumn{4}{c}{ Tabela I - Critérios para classificação de Child-Pugh } \\
\hline Encefalopatia & Ausente & Confusãomental & Coma \\
Ascite & Ausente & Moderada* & Importante** \\
Albumina & $>3,5 \mathrm{~g} / \mathrm{dl}$ & $>2,8<3,5 \mathrm{~g} / \mathrm{dl}$ & $<2,8 \mathrm{~g} / \mathrm{dl}$ \\
Bilirrubinatotal & $<2 \mathrm{mg} / \mathrm{dl}$ & $>2<3 \mathrm{mg} / \mathrm{dl}$ & $>3 \mathrm{mg} / \mathrm{dl}$ \\
Bilirrubinatotal*** & $<4 \mathrm{mg} / \mathrm{dl}$ & $>4<10 \mathrm{mg} / \mathrm{dl}$ & $>10 \mathrm{mg} / \mathrm{dl}$ \\
Tempo/Protrombina & $<4,0 \mathrm{seg}$ & $>4,0<6,0 \mathrm{seg}$ & $>6,0 \mathrm{seg}$ \\
Pontos/parâmetro & $\mathrm{I}$ & 2 & 3 \\
Pontostotais & $5-6$ & $7-9$ & $10-15$ \\
Classificação & $\mathrm{A}$ & $\mathrm{B}$ & $\mathrm{C}$ \\
\hline
\end{tabular}

*ascite compensada com uso de diurético; ** ascite descompensada apesar do uso de diurético; ${ }^{* * * *}$ na cirrose biliar

Figura I - Função neuromotora em pacientes pré-transplante hepático: força de quadríceps; equilíbrio estático; coordenação; velocidade da marcha $(\mathrm{m} / \mathrm{s})$

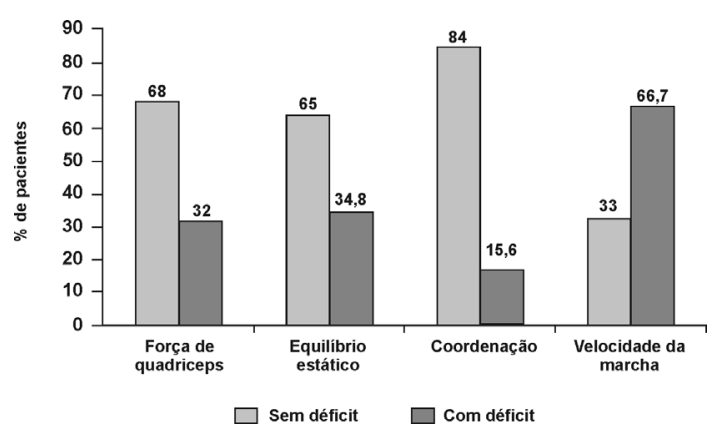

caminhada mede a distância percorrida pelo paciente, à velocidade confortável, durante seis minutos, em corredor sem obstáculos e com comprimento mínimo de $17 \mathrm{~m}$. As freqüências respiratória e cardíaca, a pressão arterial, a saturação de $\mathrm{O}_{2}$ (registro com oxímetro de pulso) e o registro de percepção à fadiga, pela escala subjetiva de Borg, são monitorados no início e no final da caminhada. O resultado do teste é estabelecido em relação a valores normais, de acordo com a equação proposta por Enright et al. (1998) ${ }^{15}$. Foi adotada a seguinte estratificação dos resultados: normal (> $90 \%$ do previsto), comprometimento leve (80\% - 90\%), moderado (60\% - $79 \%$ ) e grave ( $<60 \%$ do previsto).

$A$ independência nas atividades da vida diária e o nível de desempenho físico foram avaliados, respectivamente, pelos índices de Barthel ${ }^{18}$ e de Karnofsky ${ }^{19}$. O índice de Barthel avalia a autonomia individual para autocuidados, sendo considerada normal uma pontuação acima de 80. Oíndice de Karnofsky avalia o grau de comprometimento funcional por meio de questionário. O paciente é classificado segundo o número de pontos: 100 paciente normal, sem queixas, sem evidência de doença; 90 - atividade normal, leves sintomas de doença; 80 - atividade normal com esforço, alguns sintomas de doença; 70 - capaz de autocuidados, incapaz de atividade normal ou de trabalho; 60 - requer assistência ocasional, mas é capaz de autocuidados; 50 - requer considerável assistência e freqüentes cuidados médicos; 40 - incapacitado, requer cuidados especiais e assistência; 30 - gravemente incapacitado, indicação de hospitalização, morte não é iminente; 20 - muito doente, hospitalização necessária; e 10 - moribundo, processo fatal em progressão rápida.

$O$ estado nutricional foi avaliado com base em parâmetros antropométricos (prega cutânea do tríceps; circunferência do braço, circunferência muscular do braço), hematológico (hematócrito) e imunológico (contagem total de linfócitos). 0 percentual de adequação de cada parâmetro foi verificado com auxílio de tabelas populacionais regionais de distribuição de percentis $^{20,21}$, considerando-se o percentil 50 como sendo o valor ideal. $O$ diagnóstico nutricional foi dado pela análise dos parâmetros em conjunto, isto é, a soma dos parâmetros nutricionais em percentual de adequação dividida pelo número de parâmetros avaliados, segundo proposição inicial de Mendenhall et al. (1986) ${ }^{22}$. O valor assim obtido permitiu classificar os pacientes, segundo critérios de Blackburn et al. $(1977)^{23}$, em eutróficos (100\% de adequação), levemente desnutridos (80 $100 \%)$, moderadamente desnutridos $(60 \%-80 \%)$ e gravemente desnutridos $(<60 \%)$. No entanto, para fins de análise estatística, os graus de desnutrição moderada e grave foram agrupados.

Na análise estatística as variáveis contínuas estão descritas em médias e desvios padrão e as categóricas em distribuições de freqüência. $O$ desempenho no teste de seis minutos de caminhada, a pontuação obtida no índex de Karnofsky e o estado nutricional foram estratificados em categorias para comparação com a classificação de Child-Pugh. O teste qui quadrado foi empregado para testar as diferenças entre as distribuições de freqüência das variáveis. Os resultados foram considerados significativos quando $p<0,05$.

\section{Resultados}

\section{Avaliação funcional neuromotora}

A força muscular do quadríceps foi encontrada muito diminuída (< grau 3 no TMM) em $32 \%$ dos pacientes avaliados. $A$ força dos flexores plantares estava levemente afetada em apenas dois pacientes e nenhum paciente apresentou dificuldade para caminhar nos calcanhares. O equilíbrio estático estava prejudicado em $34,8 \%$ dos pacientes e a coordenação estava comprometida em $15,6 \%$. O valor médio da velocidade da marcha foi de $1,2 \pm 0,3 \mathrm{~m} / \mathrm{s}$ e estava reduzido em $66,7 \%$ dos pacientes (Figura I). 0 valor médio da distância percorrida no teste de seis minutos de caminhada foi de $383,8 \pm 115,4 \mathrm{~m}$. O teste foi classificado como normal em 18,2\% dos pacientes, comprometido levemente em $9,1 \%$, moderadamente em $40,9 \%$ e gravemente em 31 ,6\% (Figura 2). Em contraste, 
Figura 2 - Distribuição dos pacientes pré-transplante hepático segundo a distância percorrida no teste de seis minutos de caminhada

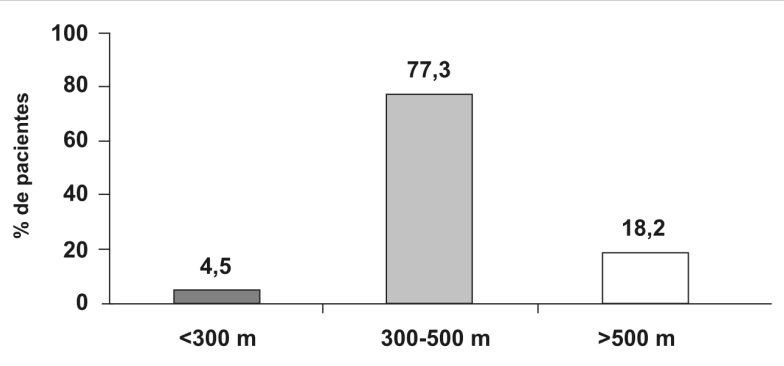

Distância percorrida em seis minutos

Figura 3 - Distribuição dos pacientes pré-transplante hepático segundo o desempenho no índice de Karnofsky

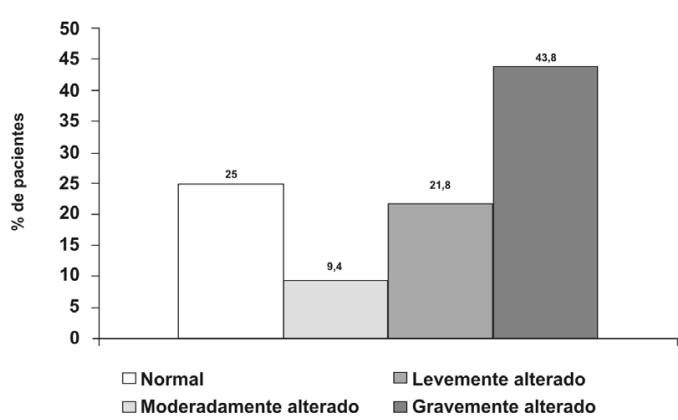

Figura 4 - Distribuição dos pacientes pré-transplante hepático quanto ao estado nutricional

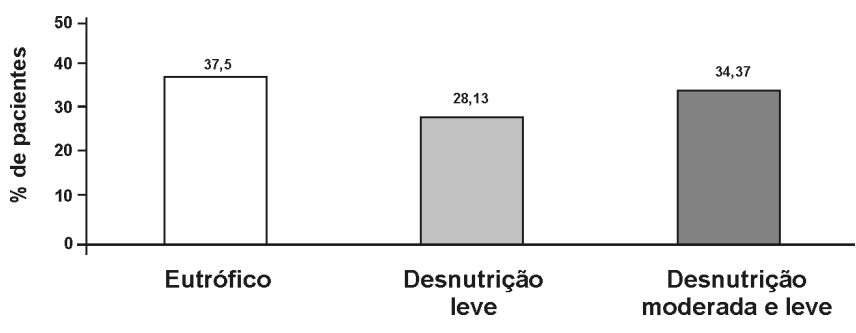

o teste "timed get up and go" foi normal em todos os pacientes do estudo. A independência funcional da vida diária, analisada pelo índice de Karnofsky, foi normal em apenas $25 \%$ dos pacientes, prejudicada levemente em $9,4 \%$, moderadamente em $21,8 \%$ e gravemente em $43,8 \%$ (Figura 3 ). Entretanto, de acordo com o índice de Barthel, todos os pacientes apresentaram pontuação acima de 80 .

\section{Avaliação nutricional}

A avaliação nutricional identificou que $37,5 \%$ dos pacientes estavam eutróficos, $28,13 \%$ apresentavam desnutrição leve e $34,37 \%$ estavam moderadamente ou gravemente desnutridos (Figura 4).

$\mathrm{Na}$ análise estatística não foi verificada associação entre o grau de comprometimento da função hepática e o estado nutricional, o teste de seis minutos de caminhada ou o índice de Karnofsky. Entretanto, a associação entreo índice de Karnofsky e o teste de seis minutos de caminhada foi altamente significante $(p<0,00 I)$.

\section{Discussão}

A desnutrição em pacientes com doença hepática crônica aguardando o transplante de fígado é freqüente ${ }^{5,24}$, tendo sido correlacionada às taxas de morbidade e mortalidade associadas ao transplante ${ }^{25}$. No entanto, sua prevalência depende da etiologia da doença e do tipo de avaliação nutricional utilizada ${ }^{26}$. No presente estudo, cerca de $2 / 3$ dos pacientes apresentavam estado nutricional comprometido, o que está de acordo com os resultados encontrados por outros autores ${ }^{27,28}$. Este comprometimento nutricional foi detectado nos três estágios da classificação de Child, portanto, sem relação com grau de comprometimento da função hepática. De modo semelhante, Campillo et al. (1997) ${ }^{24}$ encontraram, em cirróticos não-alcoólicos, alta prevalência de desnutrição, a qual foi independente da classificação de Child-Pugh. Além desse, outros estudos ${ }^{29,30}$ também observaram redução significativa nos parâmetros nutricionais em pacientes cirróticos classificados como Child A.

Embora os parâmetros clássicos de avaliação nutricional, tais como as medidas antropométricas e a determinação de proteínas plasmáticas, sejam citados como indicadores de desnutrição em pacientes com doença hepática avançada ${ }^{2,26,31}$, é reconhecido o fato de que a cirrose hepática pode alterar a maioria destes parâmetros, levando alguns autores a sugerir uma avaliação nutricional o mais abrangente possível neste tipo de pacientes ${ }^{26}$. Em busca de melhor equacionar este problema, um estudo realizado no Brasil sugeriu a utilização de parâmetros de avaliação nutricional que independam do metabolismo hepático, tais como a determinação da hemoglobina ou do hematócrito no sangue associada às medidas antropométricas, como a circunferência do braço e a prega cutânea sub-escapular ${ }^{32}$, as quais não são alteradas por sinais clínicos da doença como ascite e edema de membros inferiores. Por outrolado, parâmetros como hemoglobina e hematócrito poderiam estar alterados em caso de hipertensão portal grave acompanhada de esplenomegalia maciça e hiperesplenismo. 
Embora nossos pacientes não apresentassem pancitopenia (dados não mostrados), não podemos excluir totalmente a possibilidade de seqüestro de hemáceas pelo baço. Se de fato isto ocorreu nos pacientes com hipertensão portal e ascite, tanto o grau da ascite como a redução da hemoglobina ou do hematócrito estariam contribuindo, respectivamente, para a piora dos índices de Child-Pugh e desnutrição, e, portanto, não poderiam ser responsabilizados pela ausência de associação entre estes índices.

Os pacientes pré-transplante hepático apresentam, reconhecidamente, fraqueza e fadiga, as quais levam ao descondicionamento físico, expresso por redução da capacidade aeróbica, da força e da resistência musculares ${ }^{3,33}$. No presente estudo, encontrou-se redução da força de quadríceps e prejuízo do equilíbrio estático em cerca de 1/3 dos pacientes. A redução da força de quadríceps aliada à ausência de fraqueza muscular distal do membro inferior sugere miopatia metabólica, a qual freqüentemente acompanha a doença hepática grave? ${ }^{2}$.

A redução da capacidade física em pacientes pré-transplante hepático afeta o desempenho para as atividades da vida diária, ${ }^{6,33}$. Tem sido verificado que o teste de seis minutos de caminhada é um método de fácil aplicação e baixo custo para mensurar a função física. Este é um componente importante da qualidade de vida, porque reflete a capacidade para realizar as atividades cotidianas ${ }^{15,34}$. Beyer et $\mathrm{al}^{6}$ encontraram valores de $460 \pm 22 \mathrm{~m}$ para a distância percorrida no teste de seis minutos de caminhada em 17 pacientes que aguardavam o transplante de fígado. Após a realização do transplante, em oito dos pacientes avaliados, estes autores verificaram aumento de $27 \%$ na distância percorrida ( $563 \pm 48 \mathrm{~m})$. No presente estudo, o percurso atingido no testede seis minutos de caminhada nos pacientes prétransplantefoibastanteinferior $(383,8 \pm 115,4 \mathrm{~m})$, sugerindo maior déficit da capacidade física na população estudada. Além da baixa tolerância ao exercício, a redução da velocidade da marcha e a baixa pontuação no índice do Karnofsky confirmam este fato.

O índice de Karnofsky é uma escala de desempenho físico, que foi inicialmente desenvolvida para avaliar a capacidade física em pacientes oncológicos ${ }^{19}$, mas que tem tido seu uso ampliado para avaliação de outras doenças crônicas incapacitantes ${ }^{35}$. O resultado da aplicação deste índice, nos pacientes candidatos ao transplante de fígado deste estudo, indicou que a maioria dos pacientes necessitava de considerável assistência nas suas atividades de vida diária, bem como de freqüentes cuidados médicos.

Tanto o índice de Barthel como o teste "timed get up and go" não mostrou poder discriminatório da função neuromotora nesta população. $O$ índice de Barthel, por ser direcionado apenas para atividades básicas de autocuidados, como higiene pessoal, alimentação e controle de esfíncteres, não leva em conta outras atividades normais da vida diária, o que pode explicar a pontuação obtida pelos pacientes deste estudo.

A associação fortemente positiva entre 0 teste de seis minutos de caminhada e o índice de Karnofsky sugere que este índice possa também ser utilizado para avaliação do desempenho funcional de pacientes com doença hepática crônica à espera do transplante de fígado.

A classificação de Child-Pugh ${ }^{7}$ élargamente utilizada para avaliação da função e do prognóstico da doença hepática, principalmente nos pacientes com cirrose ${ }^{30,36,37,38}$, além de servir como um dos critérios de elegibilidade ao transplante de fígado ${ }^{39,40}$. Porém, recentemente, devido à subjetividade de alguns parâmetros $e$ limitada capacidade de discriminação desta classificação quanto ao risco de vida na lista de espera, alguns autores têm preconizado o seu emprego associado a outros testes quantitativos de função hepática ${ }^{36,37,41,42}$. Além disso, o hipermetabolismo pré-operatório e a depleção da massa celular corporal vêm sendo apontados como fatores de maior valor prognóstico para o resultado do transplante hepático do que a tradicional classificação de Child-Pugh ${ }^{31}$.

Em conclusão, a ausência de associação entre $\circ$ estado nutricional e 0 grau de disfunção da doença hepática, e desta com os testes de desempenho funcional, conforme observado em nosso estudo, confirmam que a classificação de Child-Pugh deva ser interpretada somente como uma fotografia momentânea da função hepática e não da gravidade global da doença. Outros índices devem ser incluídos na avaliação dos pacientes com doença hepática, de forma a indicar a extensão da incapacidade física e do grau de desnutrição presentes nestes pacientes.

\begin{abstract}
Agradecimentos
Os autores agradecem à agência brasileira de fomento Fundação de Amparo à Pesquisa do Estado do Rio de Janeiro-FAPERJ.
\end{abstract}

\section{SUMMARY}

Evaluation OF THE NUTRITIONAL STATUS AND PHYSICAL PERFORMANCE IN CANDIDATES TO LIVER TRANSPLANTATION

OBJECTIVE. To evaluate the physical capacity and nutritional status of patients before orthotopic liver transplantation (OLT) and to correlate these parameters to the severity of liver function.

Methods. Thirty-two patients before OTL were classified as Child-Pugh A (25\%), B (22\%) and $C(53 \%)$. Physical capacity was analyzed by the quadriceps and ankle strength, balance, coordination, gait velocity, 6 minute walk test, get up and go test, Barthel and Karnofsky indexes. Nutritional status was evaluated by using Mendenhall score and Blackburn classification.

RESULTS. Quadriceps weakness was found in $32 \%$ of the patients. Impaired balance and coordination were detected in $34.8 \%$ and $15.6 \%$ of the patients. Low physical performance was found in $72.5 \%$ of the patients (6min walk test: $31.6 \%$ severely; $40.9 \%$ moderately impaired) and $43.8 \%$ required considerable assistance when the Karnofsky index was applied. Malnutrition was found in $62.5 \%$ of the patients $34.37 \%$ moderately and severely malnourished and $28.13 \%$ mildly malnourished). Chi-square analysis showed no association between Child-Pugh classification and nutritional status, the 6min walk test and Karnofsky index. The association between the two functional tests was statistically significant $(p<0.001)$.

CONCLUSION. The results show that physical impairment and nutritional deficit were highly prevalent in the patients studied, independent of the degree of hepatic dysfunction evaluated by the traditional Child-Pugh classification. [Rev Assoc Med Bras 2003; 49(4): 424-8]

KEY wORDS: Liver transplantation. Nutritional Status. Physical performance. Child-Pugh classification. 


\section{REFERÊNCIAS}

I. Cabre E, Gassull MA. Nutritional and metabolic issues in cirrhosis and liver transplantation. Curr Opin Clin Nutr Metab Care 2000; 3 (Suppl 5): 345-54.

2. Andersen H, Borre M, Jakobsen J, Andersen $\mathrm{PH}$, Vilstrup H. Decreased muscle strength in patients with alcoholic liver cirrhosis in relation to nutritional status, alcohol abstinence, liver function, and neuropathy. Hepatology 1998; 27:1200-6.

3. Wright KC, Nicholas J], Yoman H, Robinson L. Functional assessment of liver transplantation candidates: a preliminary report. Congress Abstracts American Academy of Physical Medicine and Rehabilitation. Arch Phys Med Rehabil 1988; 69: 742.

4. Robinson LR, Switala J, Tarter RE, Nicholas JJ. Robinson LR, Switala J etal. Functional outcome after liver transplantation: a preliminary report. Arch Phys Med Rehabil 1990; 71: 426-7.

5. Selberg O, Bottcher J, Tusch G, Pichlmayr R, Henkel E, Muller MJ. Identification of high- and low-risk patients before liver transplantation: a prospective cohort study of nutritional and metabolic parameters in 150 patients. Hepatology 1997; 25: 652-7.

6. Beyer N, Aadahl M, Strange B, Kirkegaard P, Hansen BA, Mohr T, Kjaer M. Improved physical performance after orthotopic liver transplantation. Liver Transpl Surg 1999; 5: 30 I-9.

7. Pugh RN, Murray-Lyon IM, Dawson JL, Pietroni MC, Williams R. Transection of the oesophagus for bleeding oesophageal varices. Br J Surg 1973; 60: 646-9.

8. Daniels L, Worthingham $C$. Provas de função muscular. Traduzido por Nelson Gomes de Oliveira. Rio de Janeiro: Interamericana; 1972. p. 60-I. Tradução de "Muscle Testing Techniques of Manual Examination".

9. PerryJ K. Gait analysis: normal and pathological function. Thorofare: SLACK Incorporated; 1992. p. 174-5.

I0. Dyck PJ, Kratz KM, Karnes JL, Litchy WJ, Klein $\mathrm{R}$, Pach JM et al. The prevalence by staged severity of various types of diabetic neuropathy, retinopathy, and nephropathy in a population-based cohort: the Rochester Diabetic Neuropathy Study. Neurology 1993; 43 : 2345.

I I. BuchnerDM, HornbrookMC, KutnerNG, Tinetti ME, OryMG, Mulrow CDetal.. Development of the common data base for the FICSIT trials.JAm Geriatr Soc 1993; 41 : 297-308.

12. Andriacchi TP, Ogle JA, Galante JO. Walking speed as a basis for normal and abnormal gait measurements. J Biomech 1977; I0: 26 I-8.

13. Oberg T, Karsznia A, Oberg K. Basic gait parameters: reference data for normal subjects, 10-79 years of age. J Rehabil Res Dev 1993; 30: 210-23.

14. Guyatt GH, Thompson PJ, Berman LB, Sullivan MJ, Townsend M, Jones NL et al. How should we measure function in patients with chronic heart and lung disease? J Chronic Dis 1985; 38: 517-24.
15. Enright PL, Sherrill DL. Enright PL, Sherrill DL. Reference equations for the six-minute walk in healthy adults. Am J Respir Crit Care Med 1998; 158: 1384-7.

16. Mathias S, Nayak US, Isaacs B. Balance in elderly patients: the "get-up and go" test. Arch Phys Med Rehabil I 986; 67: 387-9.

17. PodsiadloD, RichardsonS. The timed "Up\&Go": a test of basic functional mobility for frail elderly persons. JAm Geriatr Soc 2000; 48: 104-5.

I8. Mahoney FI, Barthel D. Functional evaluation: The Barthel Index. Md Med J 1 965; 14:61-5.

19. Karnofsky DA, Burchenal JH. The clinical evaluation of chemotherapeutic agents in cancer. In: Macleod CM, ed. Evaluation of Chemotherapeutic Agents. I ${ }^{\text {st }}$ ed. New York: Columbia University Press; 1949: 191-205.

20. Anselmo MAC, Burini RC, Angeleli AYO, Mota NGS, Campana AO. Avaliação do estado nutricional de indivíduos adultos sadios de classe média. Ingestão energética e protéica, antropometria, exames bioquímicos de sangue e testes de imunocompetência. Rev Saúde públ., S. Paulo 1992; 26: 46-53.

21. Mathias MRC, Cervi EC, Mira LR, Curi PR, Burini RC. Estabelecimento das faixas de normalidade de variáveis hematológicas e bioquímicas de indivíduos adultos. Influência do sexo e da faixa etária. Rev Bras Pat Clin 1986; 22: 106-II2.

22. Mendenhall CL, Tosch $\mathrm{T}$, Weesner RE, Garcia-Pont P, Goldberg SJ, Kiernan T et al.VA cooperative study on alcoholic hepatitis. II: Prognostic significance of protein-calorie malnutrition. Am J Clin Nutr 1986; 43: 2 I 3-8.

23. Blackburn GL, Bistrian BR, Maini BS, Schlamm HT, Smith MF. Nutritional and metabolic assessment of the hospitalized patient. JPEN J Parenter Enteral Nutr 1977; I : I |-22.

24. Campillo B, Bories PN, Pornin B, Devanlay M. Influence of liver failure, ascites, and energy expenditure on the response to oral nutrition in alcoholic liver cirrhosis. Nutrition 1997; I3: 6|3-2|.

25. Shaw BW Jr, Wood RP, Gordon RD, Iwatsuki S, Gillquist WP, Starzl TE. Influence of selected patient variables and operative blood loss on six-month survival following liver transplantation. Semin Liver Dis 1985; 5: 385-93.

26. Le Cornu KA, McKiernan FJ, Kapadia SA, Neuberger JM. A prospective randomized study of preoperative nutritional supplementation in patients awaiting elective orthotopic liver transplantation. Transplantation 2000; 69: 1364-9.

27. Abad A, Cabre E, Gonzalez-Huix F, Gine JJ, Dolz C, XiolXet al. Influence of the nutritional status in the prognosis and clinical outcome of hospitalized patients with liver cirrhosis. Preliminary report. J Clin Nutr Gastroenterol 1987; 2: 63-68.

28. Merli M, Riggio O, Dally L. Does malnutrition affect survival in cirrhosis? Hepatology 1996; 23: $104 \mid-5$.

29. Lautz HU, Selberg O, Korber J, Burger M, Muller MJ. Protein-calorie malnutrition in liver cirrhosis. Clin Investig 1992; 70: 478-86.
30. Crawford DH, Shepherd RW, Halliday JW, Cooksley GW, Golding SD, Cheng WS et al. Body composition in nonalcoholic cirrhosis: the effect of disease etiology and severity on nutritional compartments. Gastroenterology 1994; 106: 16II-7.

3I. Cabré E, Gassul MA. Nutritional aspects of liver disease and transplantation Curr Opin Clin Nutr Metab Care 200 I; 4: 58I-99.

32. Maio R. Influência da intensidade da disfunção hepatocelular sobre os indicadores nutricionais. [dissertação de mestrado] Botucatu: Faculdade de Medicina da Universidade Estadual Paulista, 1999.

33. Campillo B, Fouet P, Bonnet JC, Atlan G. Submaximal oxygen consumption in liver cirrhosis. Evidence of severe functional aerobic impairment. J Hepatol 1990; 10: I63-7.

34. Steele B. Timed walking tests of exercise capacity in chronic cardiopulmonary illness. J Cardiopulm Rehabil 1996; 16:25-33.

35. Ifudu O, Mayers J, Matthew J, Tan Caridad C, Cambridge A, Friedman EA. Dismal rehabilitation in geriatric inner-city hemodialysis patients. JAMA 1994; 27: 29-33.

36. Vieira OM, Ribeiro-FilhoJ. Hipertensão Portal. In: Vieira OM, Chavesco CP, Manso JEF, EulálioJMR, editores. Clinica Cirúrgica: fundamentos teóricos e práticos. I ${ }^{\mathrm{a}}$ ed. São Paulo: Editora Atheneu, 2000. p 297-302.

37. LuceyMR, Brown KA, Everson GT, FungJJ, Gish R, Keefe E, Kneteman NM, Lake JR, Martin, P, Rakela J, Shiffman ML, SoS, Wiesner RH. Minimal Criteria for placement of adults on the liver transplant waiting list: a report of a national conference organized by the American Society of Transplant Physicians and the American Association for the Study of Liver Diseases. Transplantation 1998; 66 (Suppl 7): 956-62.

38. Botta, F, Giannini E, Romagnoli P, Fasoli A, Malfati $F$, Chiarbonello $B$ Testa E, Risso D, Colla G, Testa R. MELD scoring system is useful for predicting prognosis in patients with liver cirrhosis and is correlated with residual liver function: a european study. Gut 2003; 52 : 134-39.

39. Sistema Nacional de Transplantes-Ministério da Saúde 2002. http://www.saude.gov.br.

40. United Network Organ System Report 2003. http://www.unos.org.

4I. Kim WR, Poterucha JJ, Wiesner RH, LaRusso NF, Lindor KD, Petz J, Therneau TM, Malinchoc M, Dickson ER. The relative role of the Child-Pugh classification and the Mayo Natural History Model in the assessment of survival in patients with primary sclerosing cholangitis. Hepatology 1999; 29: 1643-8.

42. Kamath PS, Wiesner RH, Malinchoc M, Kremers W, Therneau TM, Kosberg CL, D'Amico G, Dickson ER, Kim WR. A model to predict survival in patients with end-stage liver disease. Hepatology 200 I; 33:464-70.

Artigo recebido: 02/12/2002

Aceito para publicação: 07/05/2003 